\title{
Image Segmentation Method of Insulator in Transmission Line Based on Weighted Variable Fuzzy C-Means
}

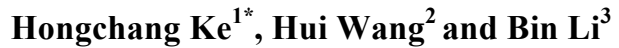 \\ ${ }^{1}$ School of Computer Technology and Engineering, Changchun Institute of Technology, Changchun 130012, China \\ ${ }^{2}$ College of Computer Science and Engineering, Changchun University of Technology, Changchun 130012, China \\ ${ }^{3}$ School of Computing Science, University of Glasgow, Glasgow, G12 8QQ, United Kingdom
}

Received 17 April 2017; Accepted 5 August 2017

\begin{abstract}
Various external environments can affect detection for insulator from the transmission line in aerial image. Segmenting the insulator from the image with the traditional image segmentation algorithm will influence the effect of detection and recognition. An image segmentation method for insulator based on ant colony classification and weighted variable fuzzy c-means (FCM) was proposed in this study to overcome the limitations of the traditional detection method for insulator in transmission line. An image enhancement method based on improved fuzzy set was introduced in the image enhancement stage. The sine membership function was used to enhance the preprocessed image according to the gray feature of insulator image. An image segmentation method based on weighted variable FCM was designed in the image segmentation stage. Different weights were defined to represent the difference between neighborhood pixels and their central pixel to divide the insulator from the transmission line. Experimental results demonstrate that the error rate of the proposed algorithm is less than 0.02 when three group insulator images (50 images per group) are randomly extracted and the signal-to-noise ratio (SNR) of the image is more than 30. The average time of image segmentation is 4.96 times lower than that of the traditional FCM. These findings indicate that the proposed algorithm fully utilizes the spatial features of the image and improves the effect and efficiency of image segmentation for insulator. The proposed method provides technical support for subsequent recognition and defect diagnosis of insulator.
\end{abstract}

Keywords: Insulator, Image enhancement, Image segmentation, Weighted variable

\section{Introduction}

The aerial transmission line, which is one of the important components of the power system, is responsible for power transmission [1]. However, high-voltage transmission lines and towers, insulators, and other accessories are usually exposed to the external environment for long periods of time, thus bearing the wind, pollution, ice, birds, and other violations. Therefore, the power sector must promptly determine the status of these attachments to reduce the occurrence of accidents, such as large areas of power outages [2-3]. Insulators are important accessories for transmission lines, which require periodic monitoring of their status. In the event of a failure, for instance, crack and filth, insulators should be immediately repaired and replaced by the power sector. Aerial images usually include a considerable amount of information, for example, transmission lines and towers. How to accurately and effectively extract the insulators from complex backgrounds is important to deliver assistance for subsequent identification and defect diagnosis [4-5].

Currently, many detection methods for insulator exist, such as observation, laser Doppler vibration, ultrasonic

\footnotetext{
*E-mail address: khch_2000@163.com

ISSN: $1791-2377$ @ 2017 Eastern Macedonia and Thrace Institute of Technology. All rights reserved. doi:10.25103/jestr.104.16
}

detection, infrared detection, and aerial methods. These methods have their own advantages and disadvantages [6-7]. However, these methods exhibit poor performance when transmission line images are affected by aerial angles, external light, rain, haze, and other factors [8-9]. Most of the detection methods for insulator in transmission line cannot be used to deal with image enhancement and image segmentation. Many detection algorithms employ the traditional image segmentation algorithm rather than the image enhancement algorithm. Many kinds of image segmentation algorithms exist, but only a few can be applied to detection for insulator in the transmission line. Traditional algorithms should be improved to effectively segment and identify the insulators.

For image enhancement and image segmentation of insulators in transmission line, this study proposed an image enhancement method based on improved fuzzy set and an image segmentation method based on weighted variable fuzzy c-means (FCM) to improve the segmentation effect and efficiency of the insulator.

\section{State of the art}

Currently, numerous studies on the detection for insulator in images with simple background exist, but only a few focus on the detection for insulator from the transmission line in 
aerial images. Most of the detection methods for insulator in transmission line use the traditional image segmentation algorithm or improved image segmentation algorithm. For example, According to the features of insulator in aerial images, Gorur [10] investigated and analyzed the traditional image segmentation algorithm and introduced the pulse coupled neural network to segment the insulator from aerial image and generalized Hough transformation to the segmented image. This method identified the insulator. Wang [11] established a mathematical model through rough image segmentation for insulator. The model identified the insulator by calculating the ratio of coordinates, center position, and effective pixels in the coarse segmenting region. Garrido [12] used the improved SUSAN edge image segmentation and scale invariant feature algorithm to extract the edge of insulator from the transmission line. However, this method cannot meet the requirements when the background is complex. Lin [13] designed the maximum entropy threshold method based on genetic algorithm to segment the insulator from the transmission line and then employed the double-structure cascade filter to refine the noise of the segmented image. Finally, the connected region identification method was used to identify the contours of the insulator string. This algorithm can extract the insulator from the aerial image in relatively simple backgrounds; however, the robustness of the algorithm should be improved in complicated backgrounds. Tong [14] employed wavelet theory to extract the features of insulator from highvoltage transmission lines and obtained the position of highvoltage lines and insulator according to feature matching. This algorithm can meet detection of low-pixel aerial image, but this algorithm is complex and its calculation time is large Katrasnik [15] developed the Ratio operator to perform edge detection on the transmission lines and insulators and segmented the insulators. However, the Ratio operator is a method specifically designed to detect linear targets, which requires that the direction of transmission line and horizontal edge of the image to be parallel. The insulator is a non-linear target; thus, the edge detection result is incomplete and a few edges are lost. $\mathrm{Li}$ [16] constructed a detection model for insulator based on the improved Canny operator according to the features of insulator. Initially, this model detected the transmission lines and the edge of insulators and then distinguished transmission lines and insulators. Moreover, this model has good detection effect because the background of image used in experiment is very simple. However, the noise immunity of this model is poor when the image background is complex. Jiang [17] combined the OTSU algorithm and wavelet theory to segment the insulator from the transmission line and then applied the elliptic feature parameters of Hough transform to design the classification criteria and identify the insulator. The algorithm realized the edge detection and recognition of insulators by calculating the elliptic parameters, but the efficiency of this algorithm is low. The preceding methods mentioned did not conduct image enhancement. Thus, the noise immunity of the algorithms is poor and the image segmentation algorithms cannot meet the complex background. In addition, these algorithms are complex and their calculation time is large.

The remainder of this paper is organized as follows. Section 3 describes the image pre-processing method of insulator in the transmission line, the image enhancement method of insulator in the transmission line, and the image segmentation method of insulator in the transmission line. Section 4 presents the experimental design and result analysis, which provided real experiments to evaluate the performance of the proposed algorithm. Section 5 summarizes the conclusions.

\section{Methodology}

\subsection{Image preprocessing of insulator in the transmission line}

First, the aerial image with insulator in transmission line is an RGB type color image, which has very high resolution and can reach up to $3072 \times 2048$ pixels. The processing efficiency for such large image is low; thus, the image should be scaled. Color images are directly converted to gray images. Then, the converted images are enhanced and segmented. The experiment provided poor results. In this study, the RGB space of the color image is converted into an HSI space, where $H$ is the hue, $S$ is the saturation, and $I$ is the intensity. The conversion process is shown in Eq. (1):

$$
\begin{aligned}
& H= \begin{cases}\theta & B \leq G \\
2 \pi-\theta & B>G\end{cases} \\
& S=1-\frac{3}{R+G+B} \min (R, G, B) \\
& I=\frac{R+G+B}{3}
\end{aligned}
$$

where $\theta=\arccos \left\{\frac{(R-G)+(R-B)}{2 \sqrt{(R-G)^{2}+(R-B)(G-B)}}\right\}, S \in[0,1]$, $I \in[0,255], R$ represents the red component, $G$ represents the green component, and $B$ represents the blue component. The HSI spatial model is close to the visual perception of color. The correlation among the three components is very small. $H$ is not sensitive to light and shadow, and $S$ has some influence on the imaging object with the change of light intensity. Moreover, $S$ can distinguish objects with different colors.

Numerous experiments showed that with the change of light intensity, a strong light leads to the value of $S$ become small. The $S$ component is selected as the gray image and transformed into $[0,255]$ pixel space.

\subsection{Image enhancement of insulator in the transmission line}

During the imaging process, the aerial images can cause image degradation or increasing noise [18]. Therefore, image enhancement is necessary. Image enhancement emphasizes or sharpens the image to facilitate the display, observation, or further analysis and processing according to the features of image, such as edges, contours, and contrast [19]. Image enhancement cannot increase the relevant information in the image, but it can increase the dynamic range of the selected features, thereby facilitating easy detection or identification of these features. The improved fuzzy set theory was proposed in this study to enhance the image.

\subsubsection{Extraction for fuzzy features of image}

First, the fuzzy features of the image are extracted by using the transformation function (membership function). The membership function is shown in Eq. (2): 
$\mu_{i j}=G\left(x_{i j}\right)=\left[1+\frac{x_{\max }-x_{i j}}{F_{d}}\right]^{-F_{e}}$

where $F_{d}, F_{e}$ is the transform coefficient, whose range is [0, 1]. $x_{\max }$ is the maximum gray value of the image, and $x_{i j}$ is the gray value of the current pixel.

\subsubsection{Correction for membership function}

The membership function is revised by the regression function of the fuzzy enhancement operator (INT), and the membership function $x_{i j}$ is transformed. The value $x_{i j}$ is increased or decreased by the crossover point; thus, a new fuzzy feature plane can be obtained by Eq. (3):

$\mu_{i j}=T^{(r)}\left(\mu_{i j}\right)=T\left(T^{(r-1)}\left(\mu_{i j}\right)\right)$

where $r=1,2,3, \ldots$, and $T^{(r)}$ is the $r$ iteration of function $T$. To achieve the purpose of contrast enhancement, INT is used to increase the membership value which is greater than 0.5 and reduce the membership value which is less than 0.5 . The result is shown in Eq. (4):

$T\left(\mu_{i j}\right)=\left\{\begin{array}{lr}2 \cdot\left[\mu_{i j}\right]^{2} & 0 \leq \mu_{i j} \leq 0.5 \\ 1-2 \cdot\left[1-\mu_{i j}\right]^{2} & 0.5 \leq \mu_{i j} \leq 1\end{array}\right.$

\subsubsection{Inverse transform for fuzzy domain}

A new gray scale is generated by using inverse transform. Then, the data can be transformed from the fuzzy domain to the spatial domain of the image, as shown in Eq. (5):

$x_{i j}^{\prime}=G^{-1}\left(x_{i j}^{\prime}\right)=x_{\max }-F\left[F_{d}\left(x_{i j}^{\prime}\right)^{\frac{1}{F_{e}}}-1\right]$

where $G^{-1}$ is the inverse transform of $G$.

\subsubsection{Image enhancement based on improved fuzzy sets}

The image enhancement method based on fuzzy theory has achieved better results than the traditional method in a few cases. However, for the aerial image, if the image is dark or the illumination is poor, then the upper limit for the gray scale of the image cannot be changed and the effect of image enhancement is not obvious. Therefore, the gray scale linear transformation based on fuzzy enhancement was introduced in this study. According to the gray features of the image, the relative gray scale of the pixel was used as the fuzzy features and the sine membership function was employed to enhance the fuzzy image, which avoided the loss of large gray features after image enhancement. The result of this process accelerated the selection of optimal parameter, ensured the quality of enhanced image, and improved the feasibility and efficiency of the algorithm. The experiment results showed that the improved fuzzy enhancement method demonstrate better enhancement effect than the traditional method with regard to the image of insulator in the transmission line.

(1) Mapping the image from the spatial to the fuzzy domain

The sine membership function is used to map the image from the spatial to the fuzzy domain, and the fuzzy feature plane $\left\{\mu_{i j}\right\}$ can be obtained. The sine membership function is defined as shown in Eq. (6): $\mu_{i j}=G\left(x_{i j}\right)=\left[\sin \frac{x_{i j}-M}{x_{\max }-M} \cdot \frac{\pi}{2}\right]^{k}$

where $\mu_{i j}$ is the gray scale of $(i, j)$ relative to the membership of the maximum gray scale, and $M$ is the condition parameter with a value of zero. According to the requirement, $M$ can be obtained between zero and the minimum gray value, and $k$ is the adjustment parameter. From Eq. (6), the range of $\mu_{i j}$ is $[0,1]$. The hard clipping of gray scale did not occur, which avoided the loss of a considerable amount of gray information.

(2) Mapping the image from the fuzzy domain back to the spatial domain

The fuzzy feature plane $\left\{\mu_{i j}^{\prime}\right\}$ can be obtained by inverse transformation, which is shown in Eq. (7):

$\mu_{i j}^{\prime}=M+\left(x_{\max }-M\right) \cdot \arcsin \left(\mu_{i j}^{\frac{1}{k}}\right) \cdot \frac{2}{\pi}$

(3) Fast selection of optimal parameters

The traditional algorithm generally uses the fuzzy enhancement method to transform the fuzzy feature plane to a new feature plane, and the new feature plane applies inverse transformation to obtain the output image. Considering that the gray scale range of the insulator in image is usually large, the transformation of the traditional algorithm does not change the upper limit of the gray scale. The linear transformation of gray scale is employed as the gray scale extension process in this study.

The function membership and the location of crossover point can be changed by adjusting the parameters $M$ and $k$ to achieve the image enhancement within different gray scales. According to a large number of experiments, the following method can be used to accelerate the selection of optimal parameters. A large lower limit gray value of the image results in considerable effects of parameter $M$ on $\mu_{i j}$. In general, $k$ is greater than $M$ considering the influence of $\mu_{i j}$. The range of $k$ is set as $\left[k_{\min }, k_{\max }\right]$, and $k$ is changed at a certain value interval to generate fuzzy image enhancement in batches. Moreover, the range of $k$ is adjusted to reduce the interval of the value, and the accuracy of $k$ can be improved.

The gray scale of the original image $f(x, y)$ is $\left[F_{\min }, F_{\max }\right]$, and the gray scale of the transformed image $f^{\prime}(x, y)$ is linearly extended to $\left[F_{\min }^{\prime}, F_{\max }^{\prime}\right]$.

Then, the linear transformation is defined by Eq. (8):

$$
f^{\prime}(x, y)=\frac{F_{\text {max }}^{\prime}-F_{\min }^{\prime}}{F_{\text {max }}-F_{\min }} \times\left[f(x, y)-F_{\text {min }}\right]+F_{\text {min }}^{\prime}
$$

where $F_{\min }, F_{\max }$ are the minimum and maximum gray scales of the original image, respectively. $F_{\min }^{\prime}, F_{\max }^{\prime}$ are the minimum and maximum gray scales of the enhanced image, respectively, $F_{\min }^{\prime} \leq F_{\min }$ and $F_{\max }^{\prime} \geq F_{\max }$.

The contrast of image can be increased after the gray scale linear transformation. The gray scale can be raised, and the effect of image enhancement is improved.

The image enhancement algorithm based on the improved fuzzy set is described as follows. 
Step 1: Input the original image $f(x, y)$. Then, read the image data from the file and count the maximum gray value $x_{\max }$ and minimum gray value $x_{\min }$;

Step 2: Set the initial value of $F_{\min }, r, F_{\min }=0, r=1$, as well as the initial value of $k_{\min }, k_{\max }, d$, then $k=k_{\min }$;

Step 3: Use Eq. (6) to compute the fuzzy feature plane of the image $\left\{\mu_{i j}\right\}$, and adopt Eq. (7) to compute the new fuzzy feature plane of image $\left\{\mu_{i j}^{\prime}\right\}$;

Step 4: Use Eq. (8) to perform the inverse transformation to obtain an enhanced image;

Step 5: Set $k=k_{\min }+d$ and repeat steps 3 to 4 until $k=k_{\max }$. Set the initial values to obtain the corresponding fuzzy enhanced image when the values of $k$ are $k_{\min }, k_{\min }+d, k_{\min }+2 d, \ldots, k_{\max }$;

Step 6: Observe the generated image according to the enhanced effect and repeat steps 3 to 5 until the condition is satisfied;

Step 7: Use gray scale transformation to increase the gray scale range of the image.

\subsection{Image segmentation of insulator in the transmission line}

\subsubsection{Theoretical basis of FCM algorithm}

The specific algorithm is expressed as follows. For a given sample of image $X=\left\{x_{1}, x_{2}, \ldots, x_{n}\right\}$, the sample set $X$ is divided into different classifications, which is $c$ times disjoint subsets $V=\left\{v_{1}, v_{2}, \ldots, v_{c}\right\}$ and $c$ is the number of classifications. Set $\mu_{i j}=\mu_{A}\left(x_{i}\right)$, which is the membership value of data $i$ that belongs to classification $j$. Thus, the constraint condition can be satisfied:

$$
\sum_{j=1}^{c} u_{i j}=1 \quad i=1,2, \mathrm{~L} n
$$

If $c_{j}$ is the center of each classification, $j=1,2, \ldots, c$, then the objective function is defined as Eq. (10):

$$
J(U, V)=\sum_{j=1}^{c} \sum_{i=1}^{n}\left(u_{i j}\right)^{m} \cdot\left\|x_{i}-c_{j}\right\|^{2}
$$

where $U$ is an 0-1 matrix of $c \times n, m$ is a constant that can control the fuzzy degree of the clustering results, which meets $m>1$. A minimum can be obtained under the conditional constraints, as shown in Eqs. (11) and (12).

$$
\begin{aligned}
c_{j}= & \frac{\sum_{i=1}^{n}\left(\mu_{i j}\right)^{m} \cdot x_{i}}{\sum_{i=1}^{n}\left(\mu_{i j}\right)^{m}} \\
\mu_{i j}= & \frac{\left(1 /\left\|x_{i}-c_{j}\right\|^{2}\right)^{1 / m-1}}{\sum_{k=1}^{c}\left(1 /\left\|x_{i}-c_{k}\right\|^{2}\right)^{1 / m-1}}
\end{aligned}
$$

The clustering process of the traditional FCM algorithm is iteration between the clustering center and membership degree. The convergence criterion is selected as the threshold method. $\varepsilon$ is the threshold, which satisfies $\varepsilon \leq 10^{-6}$. The membership value between the center of each clustering and the sample can be obtained when the algorithm is converged, and fuzzy clustering is completed. An image segmentation method based on ant colony classification and weighted variable FCM was proposed in this study to solve the random initialization problem for the clustering center of the FCM algorithm and segment the enhanced image. Then, the result of fuzzy clustering was transformed into a deterministic clustering.

\subsubsection{Image segmentation method of insulator based on} ant colony classification and weighted variable FCM

The images with insulator in transmission line include the target, background, noise, and boundary information, which are usually complex. The FCM algorithm is sensitive to initial parameters and noise, and the initialization of the clustering center is random. The FCM algorithm produces slight effects when processing on complex images. Therefore, the ant colony classification algorithm was proposed in this study to find the initial clustering center. The ant colony algorithm is global and robust, which can be used to effectively overcome the sensitivity of fuzzy clustering to initial parameters. The ant colony algorithm is a globally optimized heuristic algorithm. One important feature of the difference between the target and background is the gray scale of the pixel; thus, the gray value of the pixel is selected as the feature of clustering [20]. In addition, the boundary and noise points are frequently located where the gray scale is abrupt. The gradient of these points reflects this change, which is an important feature that reflects the difference between the boundary point and background or the region of target. Therefore, each ant can be set as the two-dimensional vector with gray features and gradient features. The clustering center is considered to be the "food source", which the ant needs to find. Image segmentation is performed by these ants with different features to search for food source.

The initial size of image $I$ is set as $m \times n, K$ ants are randomly distributed on image $I$, and each pixel of the image can be defined as a node. At the initial time, the amount of pheromone on each path is equal. Set $r$ as the cluster radius, and the weighted Euclidean distance between $X_{i}$ and $X_{j}$ is shown in Eq. (13).

$$
d_{i j}=\sqrt{\sum_{k=1}^{M} p_{k}\left(x_{i k}-x_{j k}\right)^{2}}
$$

The initial value for each element of information matrix $\tau$ is set as constant $\tau^{(0)}$. Then, $\rho_{i j}$ is the probability of node $(i, j)$ that selects the clustering centers, as shown in Eq. (14):

$$
p_{i j}^{n}=\left[\tau_{i j}\right]^{\alpha}\left[\eta_{i j}\right]^{\beta} / \sum_{(i, j) \in \Omega}\left[\tau_{i j}\right]^{\alpha}\left[\eta_{i j}\right]^{\beta}
$$

where $\Omega$ is the adjacent nodes of $(i, j), \eta_{i j}$ is the heuristic information of $(i, j), \alpha$ and $\beta$ are the relative importance of pheromone and heuristic matrices, respectively.

Then, determining heuristic information, which can be obtained by Eq. (15), is very important,

$$
\eta_{i j}=\frac{1}{Z} V_{C}\left(I_{i j}\right)
$$


where $Z$ is the normalization factor, $I_{i j}$ is the intensity value of $(i, j)$, and $V_{C}\left(I_{i j}\right)$ is the fitting function of the adjacent 16neighborhood.

When updating the pheromone matrix, multiple updates should be performed. The proposed algorithm should be updated twice.

The first update is performed after each ant performs each execution step. Each element of the pheromone matrix is updated by Eq. (16):

$$
\tau_{i j}=\left\{\begin{array}{lr}
(1-\rho) \cdot \tau_{i j}+\rho \cdot \Delta_{i j}^{(k)} & d_{i j} \leq r \\
0 & d_{i j}>r
\end{array}\right.
$$

where $\rho$ is the retention rate of pheromones, and $1-\rho$ is the volatilization rate of pheromones. The range of $\rho$ is limited between zero and one to prevent infinite accumulation of information. $\Delta$ is the remaining pheromone concentration between $i$ and $j$ by ant $k$ in this cycle.

The second update is performed after all the ants completed the movement in each step in accordance with Eq. (17):

$$
\tau^{(n)}=(1-\varphi) \cdot \tau^{(n-1)}+\varphi \cdot \tau^{(0)}
$$

where $\varphi$ is the attenuation coefficient of the pheromone.

The FCM algorithm is used to segment the image after the initial clustering center is obtained by the ant colony algorithm. The eigenvalues of adjacent pixels in the insulator image are usually the same or similar, that is, the eigenvalues among adjacent pixels in the image are similar and tare probably divided into the same cluster. Using the spatial neighborhood information of the pixels in the image is important to improve the segmentation quality and efficiency of the algorithm when image segmentation is conducted. An image segmentation algorithm based on weighted variable FCM that integrates into spatial information was proposed in this study. The algorithm completely and reasonably utilized the spatial information of the image and adopted different weights to improve the effect and efficiency of image segmentation for insulator.

To introduce the spatial constraint, the spatial weight function $h$ is defined by Eq. (18):

$h_{i}=\left(\frac{\left\|x_{i}-x_{i}^{\prime}\right\|^{2}}{\sum_{k \in \Omega}\left\|x_{i}-x_{i}^{\prime}\right\|^{2}}\right)^{-1 / c}$

where $c$ is the adjustment constant. $\Omega$ is the total number of neighborhood pixels in the weighted window, which is usually $3 \times 3$ or $5 \times 5$. $x_{i}$ is the weighted window of the central pixel, and $x_{i}^{\prime}$ is the neighborhood pixel of $x_{i}$. According to the adaptive weighting function, the smaller the value of $h_{i}$, the larger the difference between the neighborhood and central pixels. The neighborhood pixels can be possibly regarded as the noise or edge.

Then, the gray value of the center pixel in the window can be calculated, as shown in Eq. (19).

$$
g(x)=\frac{\sum_{k \in \Omega} h_{i} \cdot x_{i}^{\prime}}{\sum_{k \in \Omega} h_{i}}
$$

The membership matrix and cluster center are updated in Eqs. (20) and (21), respectively.

$$
\begin{aligned}
& \mu_{i j}=\frac{\left\|x_{i}-v_{j}\right\|^{2}+c\left(\left\|g\left(x_{i}\right)-v_{j}\right\|^{2}\right)^{-1 / m-1}}{\sum_{i=1}^{n}\left\|x_{i}-v_{j}\right\|^{2}+c\left(\left\|g\left(x_{i}\right)-v_{j}\right\|^{2}\right)^{-1 / m-1}} \\
& v_{i}=\frac{\sum_{j=1}^{n} \mu_{i j}^{m}\left(x_{i}+\alpha g\left(x_{j}\right)\right)}{(1+c) \sum_{j=1}^{n} \mu_{i j}^{m}}
\end{aligned}
$$

The proposed algorithm is described as follows.

Step 1: Set the initial values of the parameters for the ant colony algorithm and the improved FCM;

Step 2: Obtain the initial clustering center $V^{(0)}$;

Step 3: Obtain the space weight $h_{i}$ and central pixel value $g(x)$ by using Eq. (19);

Step 4: Update the membership matrix $U^{(t)}$ and cluster center $V^{(t)}$ by using Eqs. (20) and (21), respectively;

Step 5: Select an appropriate matrix norm to compare $V^{(t)}$ to $V^{(t+1)}$. If $\left\|V^{(t+1)}-V^{(t)}\right\| \leq \varepsilon$ is satisfied, then the iteration stops; otherwise, $t=t+1$, return to Step 3;

Step 6: Divide the pixels into the corresponding classification according to the maximum membership method and output segmentation result.

\section{Result analysis and discussion}

\subsection{Experimental design}

The aerial image in the experiment, which used different shooting equipment under various weather conditions from China National Grid Songyuan Electric Power Company and Jilin Electric Power Company, is randomly collected to verify the effectiveness of the algorithm. The experimental data set is composed of nine groups with a total of 450 images, and each group includes 50 images. Parts of the images have random noises. The size of the experimental image is processed to $320 \times 320$ pixels. Parts of the images are shown in Fig. 1, which contain sunny, cloudy, and rainy conditions. The test platform of the algorithm used Windows 7 Ultimate, the configuration of test $\mathrm{PC}$ is $3.2 \mathrm{GHz}$ frequency with 4 GB memory, and the algorithms are performed in MATLAB 7.0.

Experiment I randomly selected an image from each of the three groups of images. First, image preprocessing was performed by using the algorithm shown in Section 3.1, and the $S$ component of the image was obtained. Then, the traditional fuzzy set algorithm and the improved fuzzy set image enhancement algorithm proposed in Section 3.2 were used to enhance and compare the preprocessed image.

Experiment II shows the results of image segmentation for insulators in the transmission lines. The traditional FCM algorithm and the image segmentation algorithm based on ant colony classification and weighted variable FCM 
proposed in Section 3.3 were used to segment and compare the enhanced images.

Experiment III used the error probability function shown in Ref. [21] to verify the effect of the algorithm based on ant colony classification and weighted variable FCM, then tested the FCM algorithm, Ref. [21] algorithm, and the proposed algorithm. The error probability function is shown in Eq. (22):

$$
P_{e}=\sum_{i=1}^{c} \sum_{j=1}^{c} P\left(C_{i} \mid C_{j}\right) \cdot P\left(C_{i}\right)
$$

where $C_{i}$ and $C_{j}$ are the number of pixels of classification $i$ and $j$, respectively, and $c$ is the number of classifications.

Experiment IV verified the efficiency of the algorithm based on ant colony classification and weighted variable FCM, then compared it with the traditional FCM algorithm at run time.
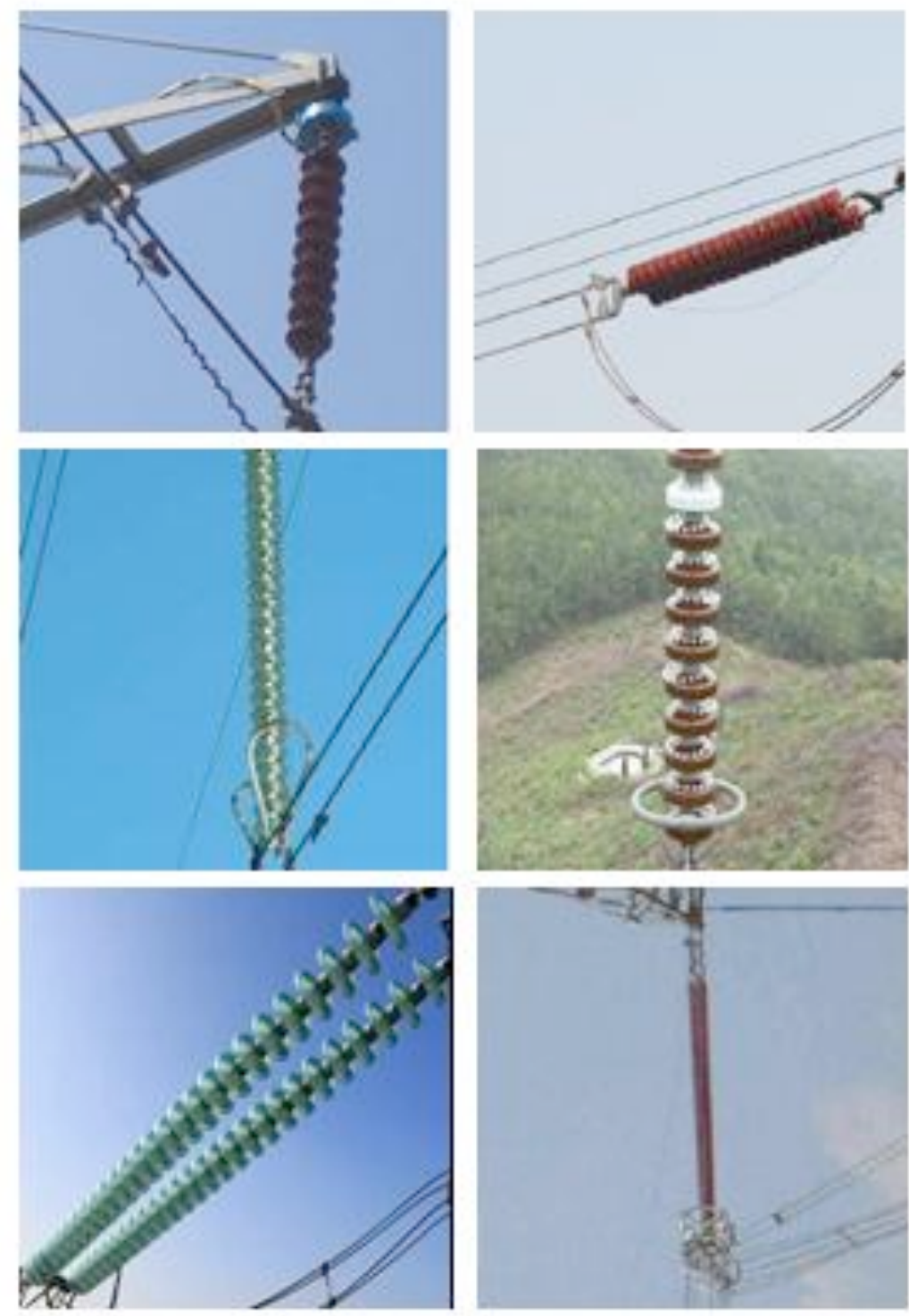

(a)

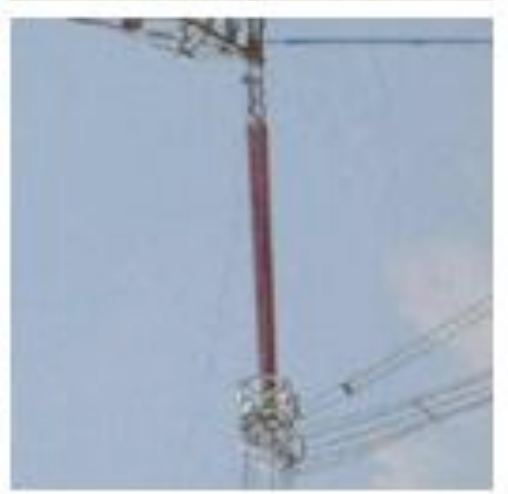

(b)
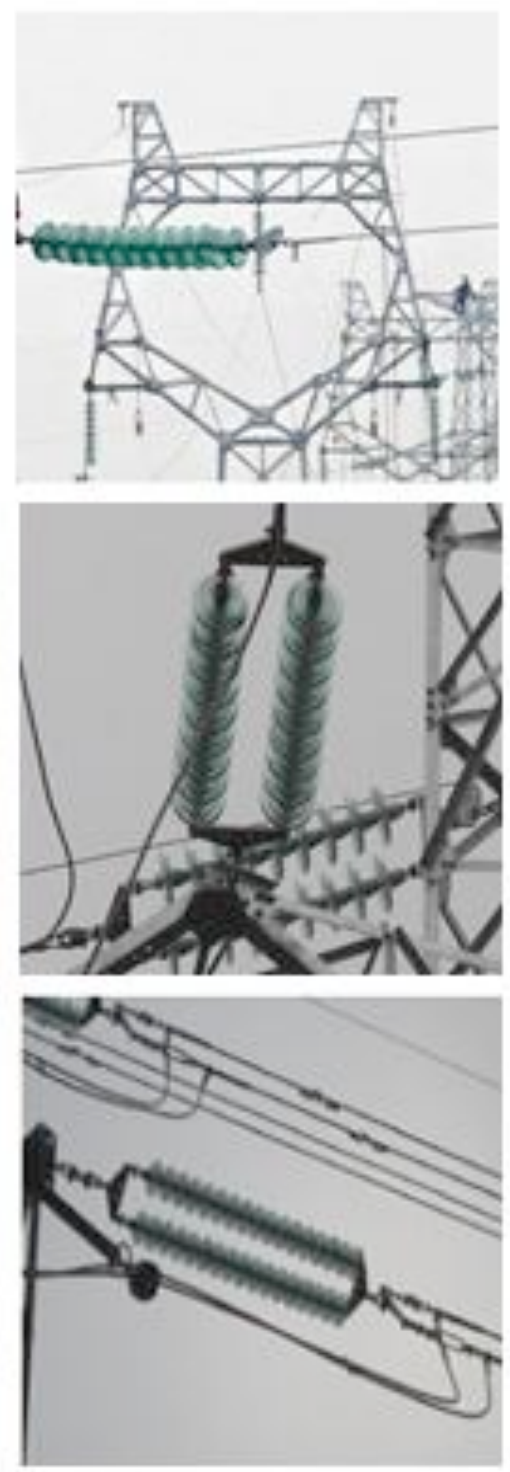

(c)

Fig. 1. Part of insulator images. (a) Sunny (b) Cloudy (c) Rainy

\subsection{Experimental results and analysis}

\section{(1) Experiment I}

The experimental results are shown in Fig. 2. Fig. 2 (a) shows the original image, while Fig. 2 (b) shows the $S$ components that RGB converted into an HSI space. The $S$ component is converted to the range of 0-255. Fig. 2 (b) shows that the $S$ component in the region of insulator is prominent, namely the saturation of the image is high. Thus, the objects with different colors can be distinguished, and the influence of most backgrounds can be removed. Fig. 2 (c) shows the results of fuzzy set enhancement with the $S$ component, and Fig. 2 (d) shows the results of proposed improved fuzzy set enhancement. The experimental results showed that $r=2, d=1$ is the best enhancement effect. Compared with the traditional image enhancement method, the proposed algorithm can overcome the limitations of the traditional image enhancement method, such as the gray scale merger, the large distortion, and the layer difference. The small range of differences can be smoothed by the proposed algorithm to achieve a good effect.

\section{(2) Experiment II}

The experimental results are shown in Fig. 3. Fig. 3 (a) shows the enhanced image. Fig. 3 (b) displays the results of 
image segmentation based on traditional FCM algorithm. Fig. 3 (c) shows the results of image segmentation using the proposed algorithm based on ant colony classification and weighted variable FCM. In the experiment, the radius of clustering center is $r=80$, and $\tau^{(0)}=0, \rho=0.85$. The number of classifications for clustering is $c=3$ and $\varepsilon=0.0001$, the maximum number of iterations is 100 , and the size of the weighted window matrix is $3 \times 3$. As shown in Fig. 3 (b), the traditional FCM algorithm can be used to segment the region

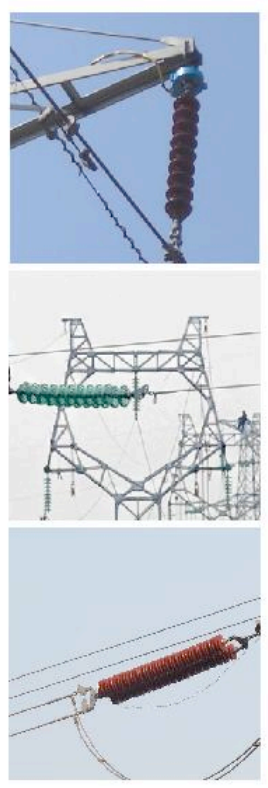

(a)
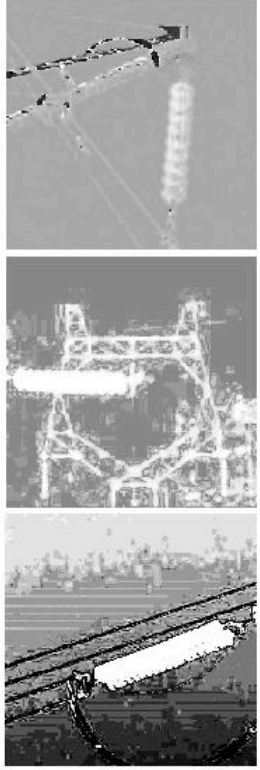

(b) of insulator, but the regions with a few of noise or the regions a small range points are not suppressed. As shown in Fig. 3 (c), the proposed algorithm can eliminate these regions.

\section{(3) Experiment III}

The experimental results are shown in Fig. 4. The error probability of the proposed algorithm is less than 0.02 when the signal-to-noise ratio $(\mathrm{SNR}) \geq 30$ is satisfied.

Fig. 2. The results of image preprocessing and image enhancement for insulators. (a) The original image (b) $S$ component of HSI (c) The enhanced image implemented by fuzzy set enhancement algorithm (d) The enhanced image implemented by proposed algorithm
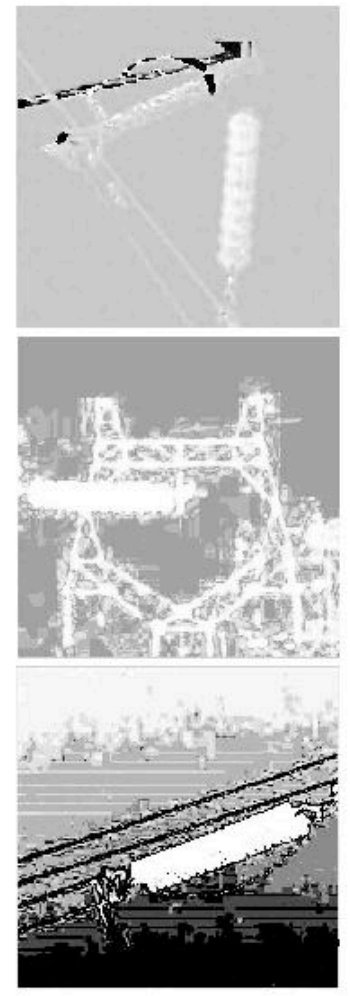

(a)
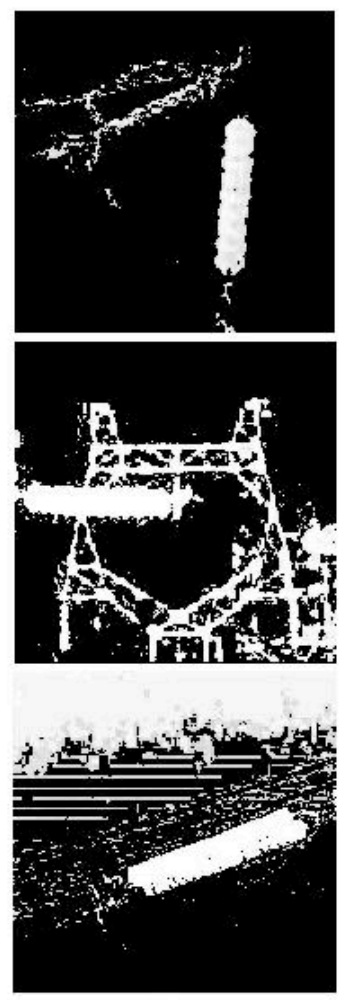

(b)
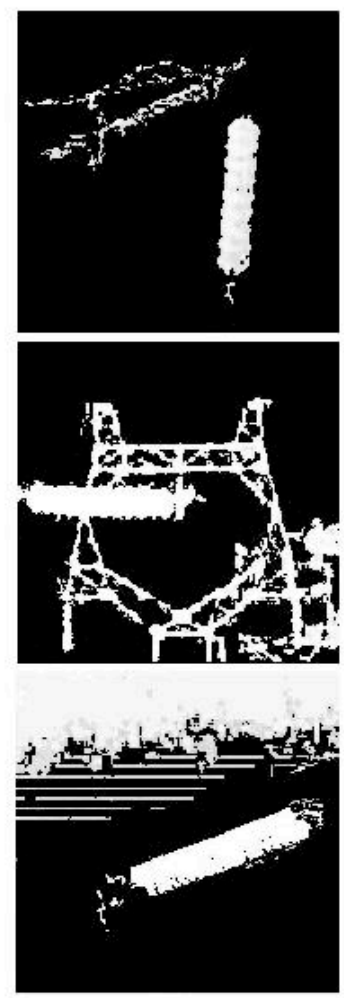

Fig. 3. The results of image segmentation for insulators. (a) The enhanced image (b) The segmented image implemented by FCM algorithm (c) The segmented image implemented by proposed algorithm 


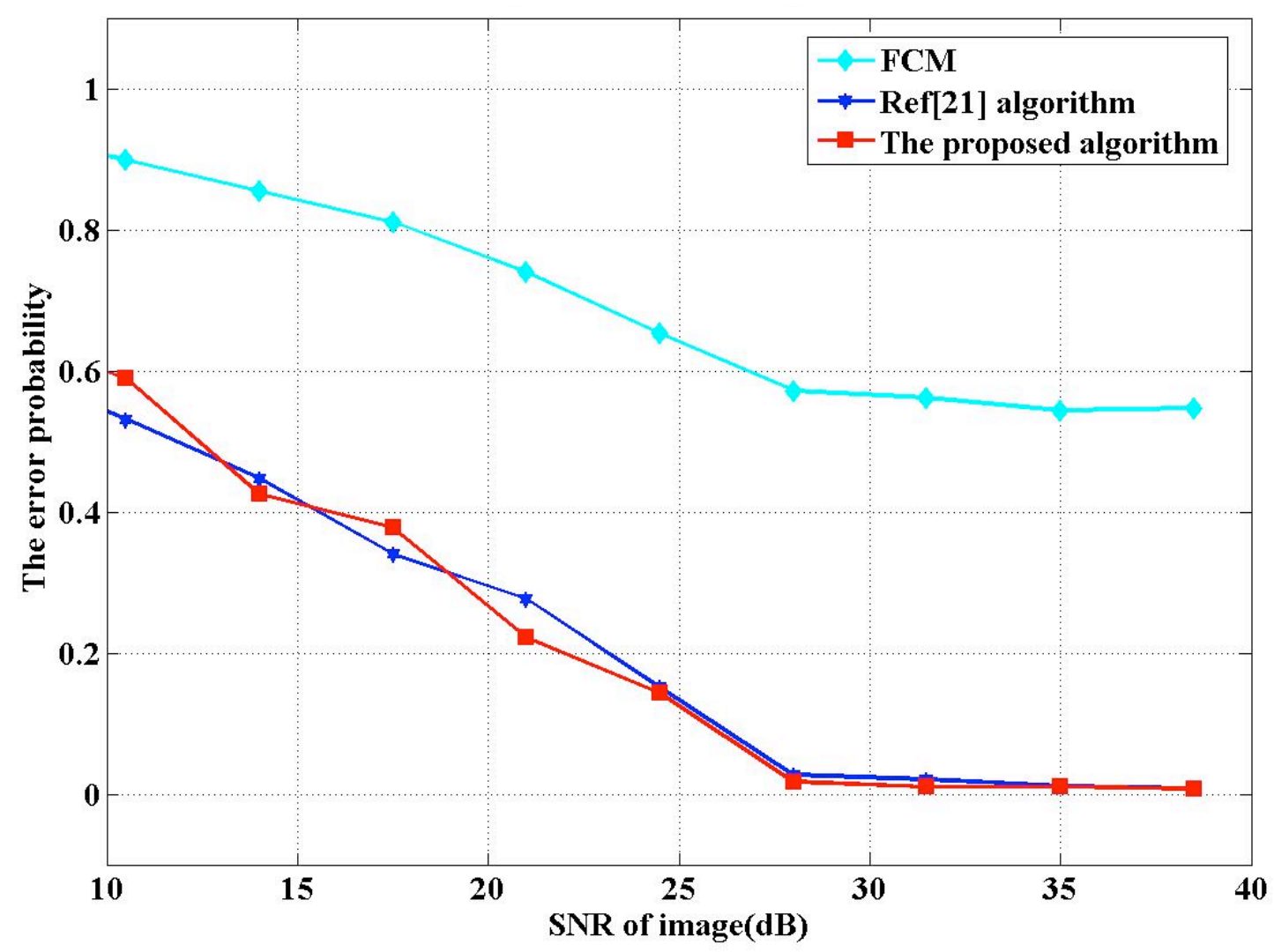

Fig. 4. Comparison results of three different algorithms

(4) Experiment IV

Compared to the operation time in Table 1, using the traditional FCM method to determine the number of clusters is very time consuming. The proposed algorithm can fully and rationally use the spatial information of the image after the colony classification algorithm to determine the clustering center. The proposed algorithm improved the efficiency of image segmentation for insulator and significantly reduced the computing time.

Tab. 1. Comparison results of operation time between the two methods

\begin{tabular}{c|c|c}
\hline Image & FCM (ms) & $\begin{array}{c}\text { Proposed algorithm } \\
(\mathbf{m s})\end{array}$ \\
\hline Fig. 2(a)-1 & 27.43 & 5.79 \\
Fig. 2(a)-2 & 56.77 & 11.88 \\
Fig. 2(a)-3 & 30.21 & 6.12 \\
\hline
\end{tabular}

\section{Conclusions}

For the detection of insulator from the transmission line in aerial image, if processing of image enhancement is not conducted or the traditional image segmentation algorithm is directly used for segmenting insulator, then the detection effect is poor. Therefore, an image segmentation algorithm for insulator in transmission line based on ant colony classification and weighted variable FCM was proposed to solve such problems. The proposed algorithm used samples from the image library of insulator in transmission line collected in the field. The following conclusions could be drawn.

(1) The sine membership function preserves the gray scale information of the enhanced image and ensures the quality of the enhanced image.
(2) The combination of ant colony and FCM algorithms can solve the random initialization problem of clustering center. The ant colony classification algorithm has global and discrete features, which are suitable for discrete digital images. This algorithm can effectively find the initial clustering center of FCM.

(3) Different weights are added to solve the low efficiency problem of FCM. The image segmentation algorithm based on weighted variable FCM can reflect the difference between the neighborhood and central pixels by using different weights, which can reduce the influence of noise or abnormal data on results of image segmentation. The proposed algorithm improved the efficiency of image segmentation.

This study considered the processes of image enhancement and image segmentation for the insulator in aerial transmission line. The image enhancement method preserved the gray information of the image, and the image segmentation method combined the ant colony classification algorithm and conducted the weight setting. The proposed method improved the effect and efficiency of the image segmentation for insulator the transmission line, which is of considerable significance to the research on subsequent recognition and defect diagnosis for insulator. Optimizing the selection of ant colony parameters in the ant colony classification algorithm to improve the efficiency of the algorithm is our next research direction.

\section{Acknowledgements}

This work was supported by: A Project Supported by the Scientific Research Fund of Jilin Provincial Education (2016310); A Project Supported by the Scientific Research Fund of Jilin Provincial Education (2016346); A Project Supported by the Scientific and Technological Planning Project of Jilin Province (20170520059JH). 
This is an Open Access article distributed under the terms of the Creative Commons Attribution Licence

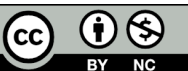

\section{References}

1. Mei, F., You, J. B., Nie, W., "Simulation and detection of photonic Chern insulators in a one-dimensional circuit-QED lattice". Physical Review A, 92(4), 2015, pp. 041805.

2. Dankert, A., Geurs, J., Kamalakar, M. V., "Room temperature electrical detection of spin polarized currents in topological insulators". Nano Letters, 15(12), 2015, pp. 7976-7981.

3. Viti, L., Coquillat, D., Politano, A., "Plasma-wave terahertz detection mediated by topological insulators surface states". Nano Letters, 16(1), 2015, pp. 80-87.

4. Werneck, M. M., Santos, D. M. D., Carvalho, C. C. D., "Detection and monitoring of leakage currents in power transmission insulators". IEEE Sensors Journal, 15(3), 2015, pp. 1338-1346.

5. Costa Jr, A. C. S., Menezes, P. C. F., Andrade, A. J. V., Silva, T., Costa, E. G., Costa, A. C. F. M., "Evaluation of current in composites PVAL/ceramics for detection of leakage current generated in electrical insulators". In: Materials Science Forum of 21st Brazilian Conference on Materials Science and Engineering, Brasilia, Brazil: TTP, 2016, pp. 308-313.

6. Yuan, C., Xie, C., Li, L., Zhang, F., Gubanski, S. M., "Ultrasonic phased array detection of internal defects in composite insulators". IEEE Transactions on Dielectrics and Electrical Insulation, 23(1), 2016, pp. 525-531.

7. Liao, S., An, J. A., "Robust insulator detection algorithm based on local features and spatial orders for aerial images". IEEE Geoscience and Remote Sensing Letters, 12(5), 2015, pp. 963-967.

8. Huang, X. N., Zhang, Z. L., "A method to extract insulator image from aerial image of helicopter patrol". Power System Technology, 34(1), 2010, pp. 194-197.

9. Lu, B. B., Liu, G. H., "Recognition of porcelain bottles crack types based on neural network". Electrical Measurement and Instrumentation, (43)492, 2006, pp. 11-15.

10. Gorur, R. S., Burnham, J. T., "Electrical performance of nonceramic insulators in artificial contamination tests. Role of resting time”. IEEE Transactions on Dielectrics and Electrical Insulation, 3(6), 1996, pp. 827-835.
11. Wang Y. L., Yan B., "Vision based detection and location for cracked insulator". Computer Engineering and Design. 35(2), 2014, pp. 583-587.

12. Garrido-Jurado, S., Muñoz-Salinas, R., Madrid-Cuevas, F. J., Marín-Jiménez, M. J. "Automatic generation and detection of highly reliable fiducial markers under occlusion". Pattern Recognition, 47(6), 2014, pp. 2280-2292.

13. Lin, J., Han, J., Chen, F., Xu, X., Wang, Y., "Defects detection of glass insulator based on color image". Power System Technology, 35(1), 2011, pp. 127-133.

14. Tong, W. G., Yan, H. J., Zhang, Q. S., "Insulator fault diagnosis based on neural network". Computer Simulation. 30(9), 2013, pp. 310-313.

15. Katrasnik, J., Pernus, F., Likar, B., “A survey of mobile robots for distribution power line inspection". IEEE Transactions on Power Delivery, 25 (1), 2010, pp. 485-493.

16. Li, Z., Liu, Y., Hayward, R., Zhang, J., Cai, J., "Knowledge-based power line detection for UAV surveillance and inspection systems". In: 23rd International Conference on Image and Vision Computing, Christchurch, New Zealand: IEEE, 2008, pp. 1-6.

17. Jiang, H. R., Jin, L. J., Yan, S. J., "Recognition and fault diagnosis of insulator string in aerial images". Journal of Mechanical and Electrical Engineering, 32(2), 2015, pp. 274-278.

18. Filho, T. M. S., Pimentel, B. A., Souza, R. M., Oliveira, A. L. I., "Hybrid methods for fuzzy clustering based on fuzzy c-means and improved particle swarm optimization". Expert Systems with Applications, 42(17), 2015, pp. 6315-6328.

19. Ke, H. C., Sun, H. B., Gao, L., Wang, H., "A video image compression method based on visually salient features". Journal of Digital Information Management, 12(5), 2014, pp. 333-339.

20. Chicco, G., Ionel, O. M., Porumb, R., "Electrical load pattern grouping based on centroid model with ant colony clustering". IEEE Transactions on Power Systems, 28(2), 2013, pp. 1706-1715.

21. Xie, M. X., Chen K., Guo, J. Z., "Research of FCM for image segmentation based on graph theory". Journal of Computer Applications. 28(11), 2008, pp. 2912-2914. 